\title{
ANÁLISIS DE LA COORDINACIÓN DE LOS BRAZOS Y LA CABEZA EN EL FLIC-FLAC EN TOURNANT, DE GRAN POSE A GRAN POSE
}

\author{
África Hernández Castillo \\ Profesora de Danza Clásica y Pedagoga Método Vaganova. \\ Investigadora independiente
}

Resumen

En este artículo se presenta la coordinación final de todas las partes del cuerpo durante la realización del paso de danza académica flic-flac en Grandes Poses. Se concluye que los resultados obtenidos en mi investigación anterior sobre la coordinación del movimiento de la cabeza en la realización del flic-flac en Pequeñas Poses, son válidos para su posterior ejecución en Grandes Poses, aunque se observan variantes en la coordinación de los brazos, la altura de la pierna de acción y el comportamiento de la cabeza en determinados momentos.

Palabras clave: FLIC-FLAC; PEQUEÑAS POSES; GRANDES POSES; PORT DE BRAS; METODOLOGÍA DANZA ACADÉMICA

\section{Abstract}

This article presents the final coordination of all body parts during the execution of the academic ballet step flic-flac in Big Poses. Taking into account the results drawn from my previous research, I can state that the coordination of the head movement for the execution of the flic-flac in Small Poses is also valid for the subsequent execution in Big Poses. Even though there are some variations in the coordination of the arms, the height of the active leg and the behavior of the head in particular moments.

Keywords: FLIC-FLAC; SMALL POSES; BIG POSES; PORT DE BRAS; METHODOLOGY OF ACADEMIC DANCE

\footnotetext{
Hernández Castil10, África. 2015. “Análisis de la coordinación de los brazos y la cabeza en el flic-flac en tournant, de Gran Pose a Gran Pose". AusArt 3 (1): 184-191. D0I: 10.1387/ausart.14760
}

\section{AUSART}




\section{INTRODUCCIÓN}

En el III Congreso Nacional Danza más Investigación celebrado en Bilbao en Noviembre de 2014, presenté una comunicación oral titulada "Análisis de la coordinación del movimiento de la cabeza en el flic-flac en tournant de Pequeña Pose a Pequeña Pose“, siendo ésta la penúltima etapa en el proceso de enseñanza-aprendizaje de este paso de danza.

Tras las conclusiones a las que llegué en dicha investigación, decidí adentrarme en la última etapa del desarrollo de este contenido analizando el flicflac en tournant de Gran Pose a Gran Pose, el cual forma parte del programa de estudios de los cursos superiores de la danza académica.

De nuevo observé que apenas existe documentación escrita, y la hallada se contradice o no llega a especificar claramente la coordinación detallada entre la cabeza, las piernas y los brazos. Además, este contenido tiene escasa presencia en el proceso de enseñanza-aprendizaje en el aula y, en caso de utilizarse, se echa en falta un análisis exhaustivo del movimiento con relación a su estructura musical, por lo que consideré imprescindible adentrarme en su investigación, indagando la precisa coordinación en su realización en Grandes Poses.

\section{OBJETIVO}

El objetivo principal ha sido comprobar que la teoría asentada para la realización del flic-flac en tournant en Pequeñas Poses es válida para su posterior ejecución a $90^{\circ}$ en face, inicialmente sin giro y luego en tournant, finalizando su desarrollo de Gran Pose a Gran Pose sin giro y después en tournant. Al mismo tiempo deberá guardar una estrecha relación con las primeras Formas (entendiendo éstas como las distintas etapas del proceso de enseñanza-aprendizaje). 


\section{METODOLOGÍA}

Para ello, se ha realizado un análisis del movimiento del paso con la adecuada coordinación de todas las partes del cuerpo, siguiendo el mismo proceso de enseñanza-aprendizaje del flic-flac en tournant en Pequeñas Poses, pero partiendo desde una posición donde la pierna de acción se encuentre a $90^{\circ}$ en lugar de a $35^{\circ}$.

Se ha considerado (al igual que en la ejecución en Pequeñas Poses) que la posición de partida para la ejecución del flic-flac sea con la pierna de base en demi-plié, estirada a pie plano o estirada en demi-pointe.

Debido a que este paso se aprenderá en un curso de nivel alto, no será necesaria su enseñanza de manera individualizada, es decir, enseñar flic y flac de manera separada, aunque sí podrá utilizarse como un movimiento conector con otro paso, o simplemente para otorgar una pincelada artística a una combinación. Tampoco será necesaria su práctica sin giro (sólo en caso de que el discente durante su aprendizaje tuviera alguna carencia que no le permitiese enfrentarse con éxito a su realización con giro), pasando directamente a su desarrollo en tournant.

\section{ANÁLISIS DEL FLIC-FLAC A 90 EN FACE Y GRANDES POSES}

\section{- Flic-Flac en tournant a $90^{\circ}$ de 2a finalizando en 2a}

Partiendo de una posición de la pierna de acción a $90^{\circ}$ y la cabeza en face, el primer bit se hará a pie plano. En el caso de estar en demipointe se descenderá a pie plano, y si nos encontramos en demi-plié se estirará la rodilla de base y nos mantendremos a pie plano. En el primer bit, en la manera en dehors los brazos se mantendrán a la $2^{\mathrm{a}}$, mientras que en la manera en dedans descenderán con un swing (oscilación) a través de una posición intermedia entre Preparatoria y $1^{\text {a }}$ para finalizar en $1^{a}$ posición. En el segundo bit se subirá a la demi-pointe realizando 1 giro, con la funcionalidad de la cabeza característica de los tours. En la manera en dehors los brazos con un swing descenderán mediante una 
posición intermedia entre Preparatoria y $1^{a}$ para finalizar en $1^{a}$ posición, y en la manera en dedans los mantendremos en $1^{a}$ posición. Se finalizará estirando de nuevo la pierna a la $2^{\mathrm{a}}$ a $90^{\circ}$ abriendo los brazos a $2^{\mathrm{a}}$ posición.

\section{- Flic-Flac en tournant a $90^{\circ}$ de 2 a finalizando delante 0 DETRÁS.}

Se llevará a cabo como el flic-flac en tournant de $2^{\mathrm{a}}$ a $2^{\mathrm{a}}$, pero finalizando con la pierna de acción delante o detrás a $90^{\circ}$ (según su realización sea en dehors o en dedans) girando la cabeza al lado.

\section{- Flic-Flac en tournant a $90^{\circ}$ desde delante o detrás terminando EN CUALQUiERA DE SUS CUATRO POSIBILIDADES EN FACE.}

Su realización seguirá las mismas pautas que de $2^{\mathrm{a}}$ finalizando delante $\mathrm{o}$ detrás, pero partiendo de una colocación de la pierna de acción delante o detrás a $90^{\circ}$, el brazo a la $2^{\text {a }}$ y la cabeza al lado. La coordinación de los brazos será la misma, pero la cabeza pasará al punto escénico 1 en el primer bit, en el segundo bit se producirá el giro, y al abrir la pierna de acción a $90^{\circ}$, colocaremos la cabeza donde lo requiera la pierna de acción.

\section{- Flic-Flac en tournant a $90^{\circ}$ de Gran Pose a Gran Pose.}

Para continuar con el desarrollo metodológico en Grandes Poses se ha partido del mismo criterio que cuando se hizo el análisis en Pequeñas Poses: realizar 1 giro en tournant de Gran Pose a Gran Pose sin considerar que, tras la realización del giro, algunas Poses no demandarán un cambio direccional del cuerpo en el espacio pero sí de Gran Pose. En cambio otras Grandes Poses sí requerirán un cambio de dirección del cuerpo en el espacio necesitando 1/4 de giro más.

Todas las Grandes Poses se identifican por tener un brazo en $3^{a}$ posición y el otro en $2^{\mathrm{a}}$ posición, por lo que habrá únicamente una forma de coordinar los brazos y la cabeza.

Cuando el flic-flac en tournant se realice en dehors, en el primer bit, que se ejecutará a pie plano, dirigiremos la cabeza hacia el punto escénico 
donde se encuentre direccionado el cuerpo, bajando el brazo que se encuentre en $3^{a}$ posición a $2^{a}$ posición.

Durante el segundo bit haremos 1 giro completo en dehors sobre la demi-pointe donde los brazos, tras dibujar una trayectoria de descenso a través de una posición intermedia entre Preparatoria y $1^{\text {a }}$, alcanzarán la $1^{a}$ posición. A ello le sumaremos la funcionalidad de la cabeza como en el tour sur le cou-de-pied (fijando la mirada en el momento del giro al punto escénico donde esté direccionado nuestro cuerpo). Y tomaremos la $2^{\mathrm{a}}$ Gran Pose al salir de nuevo la pierna a $90^{\circ}$.

Cuando el flic-flac en tournant se realice en dedans, en el primer bit que también se hará a pie plano dirigiendo la cabeza hacia el punto escénico donde se encuentre direccionado el cuerpo, bajaremos los dos brazos a través de una posición intermedia entre Preparatoria y $1^{a}$ hasta alcanzar la $1^{\text {a }}$ posición. Durante el segundo bit ejecutaremos 1 giro completo en dedans sobre la demi-pointe, manteniendo los brazos en $1^{a}$ posición y haciendo uso de la cabeza como en el tour sur le cou-de-pied (fijando la mirada en el momento del giro al punto escénico donde esté direccionado nuestro cuerpo). $\mathrm{Y}$ adquiriremos la $2^{\mathrm{a}} \mathrm{Gran}$ Pose al salir de nuevo la pierna a $90^{\circ}$.

\section{ESTRUCTURA MUSICAL DEL FLIC-FLAC}

A continuación se plasma un esquema general del análisis del movimiento en relación con la estructura musical en compás de 4/4 y en compás de 2/4.

La velocidad del metrónomo es la que nos permitirá realizar este análisis teniendo en cuenta los tiempos fuertes de cada compás. A la vez variará la velocidad dependiendo en qué ejercicio se incluya el flic-flac (grand battement, developpé, rond de jambe en láir,...)

\section{- En compás de 4/4. Negra $=90$.}

Posición de inicio: $5^{\mathrm{a}}$ posición.

Compases de preparación: 2 compases de 4/4. 
En el tiempo 4 del segundo compás saldrá la pierna a $90^{\circ}$. Compás del ejercicio: 1 compás de 4/4.

En el tiempo 1 haremos Flic. En el tiempo 2, Flac. En el 3, extenderemos la pierna a la altura de $90^{\circ}$, y en el tiempo 4 mantendremos la posición.

- En compás de 2/4. Negra $=90$.

Posición de inicio: $5^{\mathrm{a}}$ posición.

Compases de preparación: 4 compases de 2/4.

En el tiempo 1 del cuarto compás saldrá la pierna a $90^{\circ}$.

En el tiempo 2 del cuarto compás, en la primera corchea realizaremos Flic y en la segunda corchea Flac.

Compases del ejercicio: 1 compás de 2/4.

En el tiempo 1 del compás, en la primera corchea extenderemos la pierna a la altura de $90^{\circ}$, y en la segunda corchea mantendremos la posición.

En el tiempo 2 realizaremos flic-flac.

Se observa que el flic-flac en tournant a $90^{\circ}$ realizado en compás de $2 / 4$ dependiendo de con qué paso se combine, se realizará como se ha descrito arriba, o el movimiento flic-flac podrá pasar a realizarse en el tiempo 1 del compás del ejercicio y la extensión de la pierna a $90^{\circ}$ sucedería en el tiempo 2 del compás del ejercicio.

También se ha investigado si existen las mismas posibilidades de ejecución del flic-flac en tournant en Grandes Poses que en Pequeñas Poses, llegándose a la conclusión de que efectivamente, al igual que en Pequeñas Poses, hay 8 diferentes posibilidades de realización en la barra (4 en dehors y 4 en dedans), y 14 en el centro ( 7 en dehors y 7 en dedans) en Grandes Poses. Asimismo coinciden los cambios o no cambios de dirección del cuerpo en el espacio después del giro tanto en la realización del flic-flac en dehors y en dedans en Pequeñas y Grandes Poses.

Durante la realización del flic-flac en pequeñas Poses habrá un allongé antes del primer bit en aquellas Poses que empiecen con uno de sus brazos en $1^{\text {a }}$ posición. Se constata por lo tanto que en Grandes Poses, no existirá ese allongé por encontrarse el brazo en $3^{\text {a }}$ posición. 
A diferencia del flic-flac en Pequeñas Poses cuya realización se estipuló de dos maneras diferentes dependiendo de la posición de los brazos en su Pose inicial y final, y de sus diferentes posibilidades de coordinación, se sintetiza una única manera de realizarlo en Grandes Poses porque todas consisten en tener un brazo en $3^{\mathrm{a}}$ posición y el otro en $2^{\mathrm{a}}$.

\section{CONCLUSIONES}

Retomando el objetivo principal, se concluye que la coordinación del movimiento de la cabeza durante el giro en la realización del flic-flac en tournant en Pequeñas y Grandes Poses será la misma, así como el uso de las diferentes direcciones del cuerpo en el espacio y el momento en el que se encontrará el cuerpo direccionado en cada movimiento del flic-flac, sea este ejecutado en dehors como en dedans. La funcionalidad de la cabeza establecida nos seguirá indicando que donde dirijamos la cabeza en el primer bit es donde finalizará la posición de la cabeza al adoptar la segunda Pose en las que haya un cambio de dirección del cuerpo en el espacio.

Sin embargo sí que se han observado diferencias en la trayectoria de los brazos, en la posición inicial de la Pose, en el primer movimiento del flic-flac (flic) y en la adquisición de la $2^{\text {a }}$ Pose debido a las diferencias que las caracteriza y al distinto comportamiento de los brazos durante sus port de bras. El segundo movimiento del flic-flac (flac) se ejecuta de la misma manera tanto en las Pequeñas como en las Grandes Poses.

Aunque parezca una evidencia, considero pertinente remarcar que existe una gran diferencia en la altura de la pierna de acción tanto en el inicio del Paso en su primera Gran Pose $\left(\mathrm{a} 90^{\circ}\right.$ ) como en su Pose final (también a $90^{\circ}$ ), ya que en la bibliografía consultada no se especifica.

\section{Referencias}

Hernández Castillo, África. 2014. La danza académica y su metodología: Nivel Medio I. Valencia: Mahali

Kostrovitskaya, Vera \& Alexei Pisarev. 1995. School of Classical Dance. Authoriced translation by John. Barker. London: Dance Books 
Tarassow, Nicolai I. 1994. Klassischer Tanz: Die Schule des Tänzers. Berlin: Henschel

Vaganova, Agripina. 1945. Las bases de la Danza Clásica. Trad. M. Ourvantzoff Buenos Aires: Centurión

Zsuzsa, Lőrinczné Merényi. 1993. A Klasszikus Balett Módszertana. Budapest: Nemzeti Tankönyvkiadó

(Artículo recibido 12-03-15; aceptado 09-06-15) 